\title{
Application of Liquid Cell-TEM in Hydration Reactions of Nano Portland Cement
}

\author{
Peng Dong ${ }^{1}$, Ali Allahverdi ${ }^{2}$, Carmen M. Andrei ${ }^{1}$ and Nabil D. Bassim ${ }^{1}$ \\ 1. Department of Materials Science and Engineering and Canadian Centre for Electron Microscopy, \\ McMaster University, Hamilton, ON, Canada \\ 2. School of Chemical Engineering, Iran University of Science and Technology, Narmak, Tehran, Iran
}

Liquid cell in-situ microscopy can provide new insight into the hydration behavior of Portland cement and new type of concretes [1] and can ultimately provide insight into reaction kinetics as a function of initial chemistry. This initial work uses liquid cell transmission electron microscopy (LC-TEM) technology to study hydration reactions and microstructure formation in a well-characterized dilute slurry of nano Portland cement in real time and at high resolution as a starting point for studying new cement formulations. To perform these experiments at electron transparency conditions, the particle size of ordinary Portland cement was reduced through ball milling in the presence of ethanol. To initiate the reaction, nano Portland cement powder was mixed with water at a w/c ratio of 15 and sonicated for 5 min to effectively disperse cementitious nano particles. The real-time hydration was studied using bright field (BF) TEM (ThermoFisher-FEI Titan, $300 \mathrm{keV}$ ) imaging using a Protochips liquid cell holder with a miniaturized liquid cell, which confines the samples in between two silicon nitride membranes. Experiments were conducted over the span of three hours to observe hydration products and their morphology.

Similarly, to known Portland cement reactions, early age needle-like ettringite $\left(\mathrm{Ca}_{6} \mathrm{Al}_{2}\left(\mathrm{SO}_{4}\right)_{3}(\mathrm{OH})_{12} \cdot 26 \mathrm{H}_{2} \mathrm{O}\right)$ crystals were observed about 2 hours into the experiment. These crystals appeared randomly distributed. Ettringite also acted as nucleation sites for precipitation of calcium silicate hydrates (C-S-H), as showed in Fig. 1 (a). With the progress of hydration reactions, ettringite crystals were consumed and, at the same time, C-S-H precipitation on the internal surface of the upper chip window of the liquid cell was observed as shown in Fig. 2(a). Unlike disordered C-S-H precipitate formations inside water, the C-S-H precipitates on the upper chip window showed ordered morphology upon their gradual growth. As Fig. 2(b) shows after about 10 min of growth, the C-S-H precipitates on SiN membrane displayed a center-oriented flower-like morphology. The flower-like C-S-H deposits increased in size and coalesced with time and the radially oriented structure cracked from the center to the edges as displayed in Fig. 2 (c). To clarify the formation of C-S-H precipitates both inside water and on the $\mathrm{SiN}$ window surfaces, an area was chosen and imaged at time intervals of 8 min as presented in Fig. 3(a) and (b). The sharp features show C-S-H precipitates formed on the upper chip window while those with blurry outlines were in the liquid or lower chip window. It was observed that round-shape precipitates nucleated and grew individually until small precipitates coalesced with large ones or connected to form a network. This is ascribed to the reduction of the surface energy of reaction.

This study confirms a first step in measuring cement hydration reactions and microstructure formation at nano scales in real time and in liquid. Real-time imaging of dissolution of cement particles in water, ettringite crystal formation and disappearance, C-S-H formation and precipitation match the real-world sequence of Portland cement hydration, although with very different kinetics. This is due to precursor particle size effects and beam-sample interactions. Studies will be undertaken to further elucidate these phenomena. 
References:

[1] Ross, Frances M. Science 350 (2015). p. 6267: aaa9886.
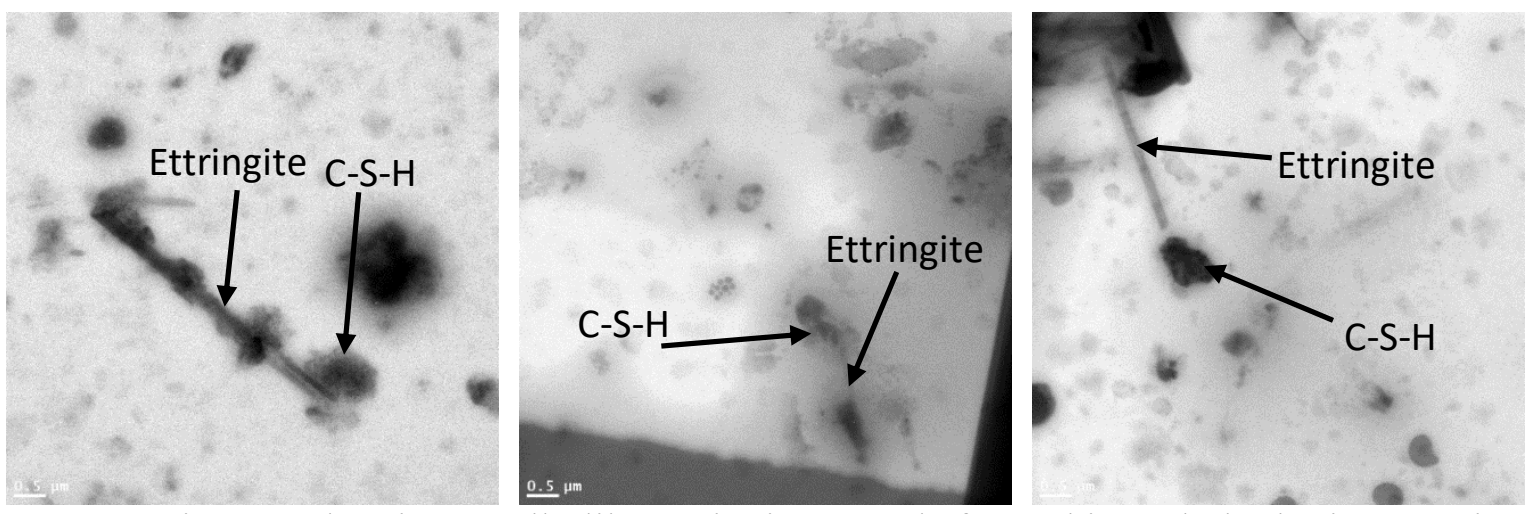

Figure 1. BF images showing needle-like ettringite crystals formed in early hydration reactions at (a) 1h41min, (b) 1h44min, and (c) $1 \mathrm{~h} 47 \mathrm{~min}$

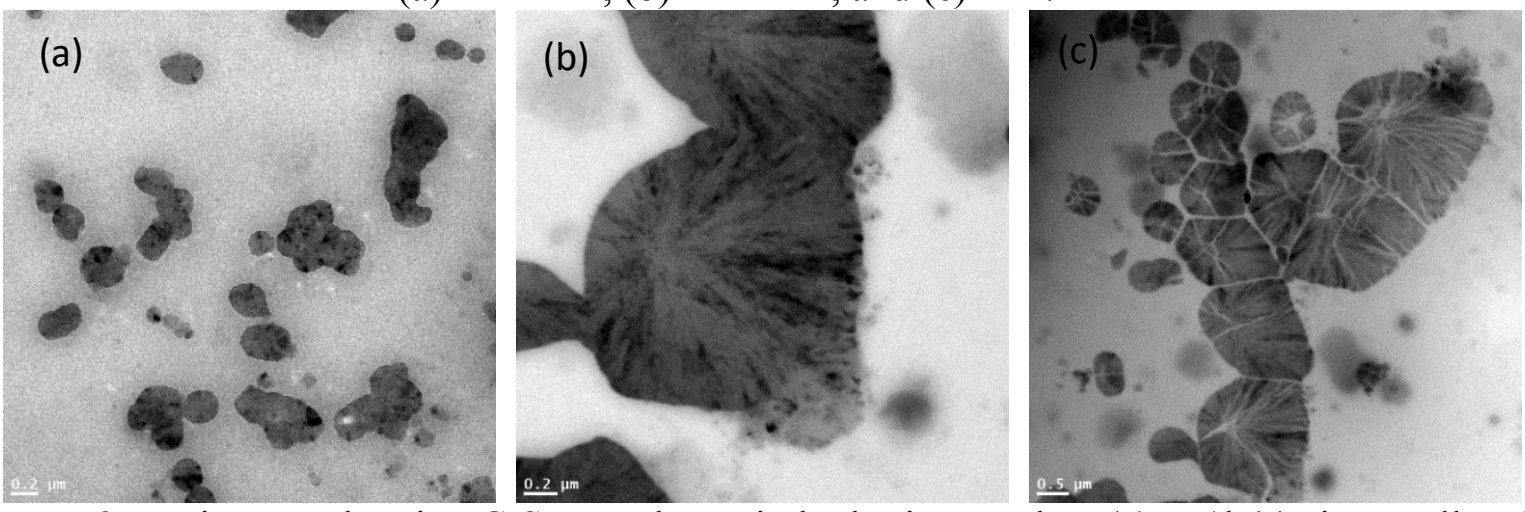

Figure 2. BF images showing C-S-H as the main hydration product (a) at $1 \mathrm{~h} 44 \mathrm{~min}$, small welldistributed and coalesced C-S-H precipitates, (b) at 1h54min, center-oriented flower-like C-S-H precipitates, and (c) at $2 \mathrm{~h} 14 \mathrm{~min}$, cracked microstructure of $\mathrm{C}-\mathrm{S}-\mathrm{H}$ precipitates
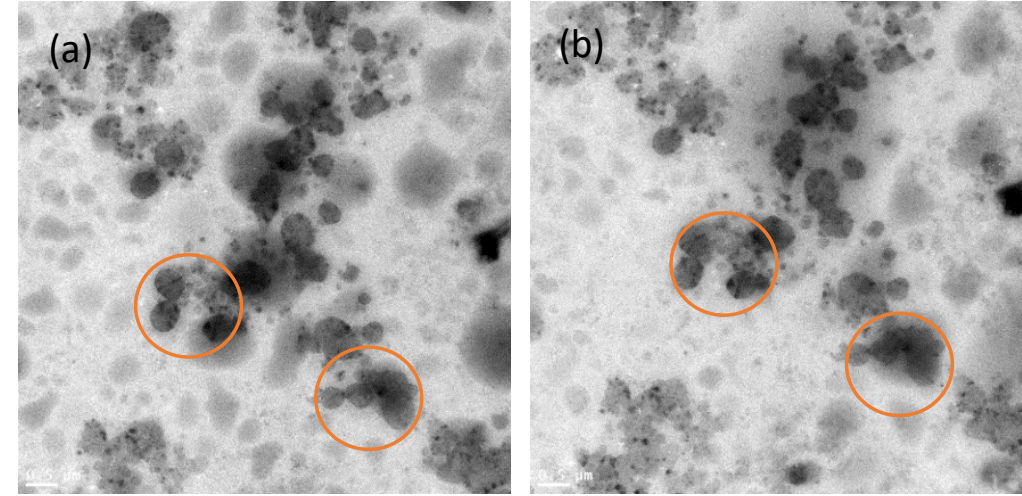

Figure 3. BF images showing agglomeration of $\mathrm{C}-\mathrm{S}-\mathrm{H}$ precipitates with time (a) at $2 \mathrm{~h} 31 \mathrm{~min}$ and (b) $2 \mathrm{~h} 39 \mathrm{~min}$ 